\title{
The transcriptomic signature of obligate parthenogenesis
}

3 Department of Biology, University of Texas at Arlington, Arlington, Texas, USA 76019

4 *Corresponding author: Sen Xu, 501 S. Nedderman Dr, Arlington, Texas, 76019 USA. Phone:

5 812-272-3986. Email: sen.xu@uta.edu.

6 Key words: Daphnia pulex, Daphnia pulicaria, meiosis, gene expression, hybrid genetic

7 incompatibility

8 Running title: Underdominance and the origin of asexuality

\section{Author contributions}

10 SX designed the experiments. SX, TH, and MS performed experiments, analyzed data, wrote the

11 manuscript. 


\section{Abstract}

14 Investigating the origin of parthenogenesis through interspecific hybridization can provide

15 insight into how meiosis may be altered by genetic incompatibilities, which is fundamental for

16 our understanding of the formation of reproductive barriers. Yet the genetic mechanisms giving

17 rise to obligate parthenogenesis in eukaryotes remain understudied. In the microcrustacean

18 Daphnia pulex species complex, obligately parthenogenetic (OP) isolates emerged as

19 backcrosses of two cyclically parthenogenetic (CP) parental species, D. pulex and D. pulicaria,

20 two closely related but ecologically distinct species. We examine the genome-wide expression in

21 OP females at the early resting egg production stage, a life-history stage distinguishing OP and

$22 \mathrm{CP}$ reproductive strategies, in comparison to $\mathrm{CP}$ females of the same stage from the two parental

23 species. Our analyses of the expression data reveal that misregulated genes (underdominant and

24 overdominant genes) are abundant in OP isolates, suggesting widespread regulatory

25 incompatibilities between the parental species. More importantly, underdominant genes (i.e.,

26 genes with expression lower than both parentals) in the OP isolates are enriched in meiosis and

27 cell-cycle pathways, indicating an important role of underdominance in the origin of obligate

28 parthenogenesis. Furthermore, metabolic and biosynthesis pathways enriched with overdominant

29 genes (i.e., expression higher than both parentals) are another genomic signature of OP isolates. 


\section{Introduction}

31 Understanding the origin and evolutionary consequences of obligately asexuality (i.e.,

32 parthenogenesis) in eukaryotes is critical for deciphering why these lineages are evolutionarily

33 short-lived (Maynard Smith 1978; Bell 1982; Otto 2009). It is well known that the evolutionary

34 consequences of obligate asexuality mainly result from the evolutionarily negligible amount of

35 meiotic recombination in asexual genomes. These include the inability to rapidly adapt due to

36 reduced efficiency of selection (Colegrave 2002; Colegrave et al. 2002; Kaltz and Bell 2002;

37 Poon and Chao 2004; Goddard et al. 2005; Cooper 2007; Kosheleva and Desai 2018), the

38 irreversible accumulation of slightly deleterious mutations (i.e., Muller's ratcher, Muller 1964;

39 Felsenstein 1974), and the mutational meltdown of asexual populations/lineages (Gabriel et al.

40 1993; Lynch et al. 1993).

However, the genetic mechanisms underlying the origin of parthenogenetic species remain understudied, despite its potential for informing us about the evolutionary dynamics of

43 asexual taxa. In eukaryotes, obligate parthenogens originate from sexually reproducing ancestors

44 through means of spontaneous mutations, hybridization between closely related sexual lineages,

45 and parasitic infections (Simon et al. 2003). During this sexual-asexual transition, meiosis is

46 altered (e.g., becoming a mitosis-like cell division) to produce chromosomally unreduced

47 gametes. The cytological modifications of meiosis in parthenogens are well studied and differ 48 dramatically between lineages (Stenberg and Saura 2009; Neiman et al. 2014). For example,

49 some lineages engage in apomixis in which the reductional phase of meiosis is skipped to

50 produce unreduced eggs, while others undergo automixis in which the fusion of meiotic products

51 restores the parental ploidy level (Stenberg and Saura 2009). However, due to a lack of

52 understanding of the genetic and molecular mechanisms underlying germline cell division in

53 parthenogens, it remains unknown how these cytological modifications arise.

54 We suggest that the efforts in resolving this knowledge gap, although still rare, will have

55 implications beyond understanding the evolution of asexuality. For example, understanding how

56 asexuality can originate through hybridization can provide us insights into how meiosis can be

57 disrupted by inter-specific genetic incompatibilities, which is essential to our understanding of

58 how reproductive barriers may arise during speciation. In speciation research, the Dobzhansky-

59 Muller model of hybrid incompatibility posits that genetic elements in two diverging lineages, 
60 when placed in the same genetic background, may negatively interact and result in fitness

61 reduction and sterility in hybrids (Dobzhansky 1937; Muller 1940). Substantial empirical

62 evidence supporting Dobzhansky-Muller incompatibilities has emerged over the years (Rawson

63 and Burton 2002; Barbash et al. 2003; Presgraves 2003; Mack and Nachman 2016). However,

64 obligate parthenogens with hybrid origin have not been explicitly considered under this

65 theoretical framework (but see Janko et al. 2018).

66 Compared to other modes of origin, hybridization is attributed for the largest number of

67 obligate parthenogens by far, with nearly all vertebrate parthenogens having hybrid ancestry

68 (Avise 2015). It is still controversial whether hybridization directly leads to parthenogenesis in

69 the $F_{1}$ generation (Kearney et al. 2009), as only a few attempts succeeded in generating

70 parthenogenetic $F_{1}$ s by crossing identified parental lineages found in nature (e.g., Schultz 1973;

71 White et al. 1977; Hotz et al. 1985; Janko et al. 2018). Also, some obligate parthenogens (e.g.,

72 Daphnia) are backcrosses derived from complex introgression events (Xu et al. 2013; Xu et al.

73 2015). Because parthenogenetic hybrids are largely incapable of backcrossing with parental

74 lineages, we argue that obligate parthenogenesis in hybrids is analogous to hybrid sterility in

75 reducing gene flow between diverging lineages. Therefore, identifying the genetic elements

76 involved in the hybrid origin of obligate parthenogenesis would greatly expand our

77 understanding of the genetics of hybrid incompatibility.

78 In this work, we examine whether gene expression plays a role in the hybrid origin of 79 obligate parthenogenesis in the freshwater microcrustacean Daphnia pulex species complex. It

80 has long been recognized that differences in gene expression are an important source of 81 phenotypic changes (Wray 2007; Stern and Orgogozo 2008). Gene expression has been 82 examined in various asexual taxa to probe the possible causes of parthenogenesis (Gallot et al.

83 2012; Hanson et al. 2013; Srinivasan et al. 2014). Nonetheless, for hybrid obligate parthenogens

84 it remains unclear how their gene expression varies relative to the parental species, whether any

85 significant expression changes affect germline cell division, and how regulatory divergences 86 contribute to expression changes.

87 We address these issues using obligately parthenogenetic (OP) Daphnia that are 88 backcrosses of two parental species, the cyclically parthenogenetic (CP) D. pulex and D. 89 pulicaria, members of the $D$. pulex species complex. Daphnia typically reproduces by cyclical 
90 parthenogenesis (Figure 1A). Under favorable environmental conditions females produce

91 directly developing embryos through parthenogenesis, generating genetically identical daughters

92 (barring de novo mutations). However, in unfavorable conditions (e.g., food shortage), some

93 asexual broods become males through environmental sex determination, and females switch to

94 producing haploid eggs. Sexual reproduction between the two produces diapausing, fertilized

95 embryos deposited in a protective case (i.e., ephippium). Interestingly, many populations in the

96 northeast of North America reproduce by obligate parthenogenesis (Figure 1B), i.e., reproducing

97 by parthenogenesis in favorable conditions, but also producing ephippial resting embryos by

98 parthenogenesis in deteriorating conditions (Hebert et al. 1993).

It should be noted that the parthenogenesis of both directly developing embryos and 101 segregation of homologous chromosomes occurs and anaphase I is not followed by formation of 102 daughter cells, i.e., no cytokinesis (Zaffagnini and Sabelli 1972; Hiruta et al. 2010). Therefore, 103 we hypothesize that in hybrid OP Daphnia isolates the anaphase promotion complex and spindle 104 checkpoint assembly, which play critical roles in chromosome segregation and cytokinesis 105 (Cooper and Strich 2011; Gorbsky 2015), are affected by aberrant gene expression due to 106 regulatory incompatibility between the parental species, CP D. pulex and D. pulicaria.

Previous work has revealed that these OP isolates originated through repeated 108 backcrossing between the parental species ( $\mathrm{Xu}$ et al. 2013; Xu et al. 2015). Genome-wide 109 association mapping showed that the microsatellite and SNPs alleles associated with OP 110 (concentrated on chromosomes 8 and 9) are only present in D. pulicaria (a species exclusively 111 inhabiting permanent lake habitats, see below), suggesting that a historical introgression event 112 led to the origin of OP (Lynch et al. 2008; Xu et al. 2015).

113 Several lines of evidence strongly suggest ecological divergence and genetic 114 incompatibility between these two parental species. As members of the D. pulex species complex, 115 CP D. pulex and D. pulicaria are estimated to have started divergence from 800,000 - 2,000,000 116 years ago (Colbourne and Hebert 1996; Omilian and Lynch 2009; Cristescu et al. 2012). These 117 two species are morphologically similar (Brandlova et al. 1972) but occupy distinct, overlapping 118 freshwater habitats in North America. Daphnia pulex mostly lives in ephemeral fishless ponds, 119 whereas D. pulicaria inhabits stratified permanent lakes. These two species show clear 
120 physiological differences (Dudycha and Tessier 1999; Caceres and Tessier 2004a, b; Dudycha

121 2004), indicating strong local adaptation and divergent selection in their distinct habitats.

122 Although D. pulex and D. pulicaria can still generate fertile $\mathrm{CP} \mathrm{F}_{1}$ offspring in laboratory 123 crossing experiments (Heier and Dudycha 2009), some initial evidence points to the presence of

124 both incomplete prezygotic and postzygotic isolation. The prezygotic barrier lies in the effects of

125 photoperiods in triggering sexual reproduction in these two species (Deng 1997), with D. pulex

126 switching to sexual reproduction at long day hours (16 hours/day) and D. pulicaria switching to

127 sex at short day hours (10 hours/day). On the other hand, postzygotic barriers likely exist as

128 interspecific crosses have lower survival and hatching success than conspecific crosses (Chin et 129 al. 2019).

130 Despite these reproductive barriers, ample opportunities exist for interspecific 131 introgression between D. pulex and D. pulicaria when ecological barriers break down (Millette 132 et al. 2020) and phenotypic changes consequently emerged, strongly indicating the widespread 133 presence of genetic incompatibility between them. In addition to obligate parthenogenesis caused 134 by the introgressed $D$. pulicaria alleles in a CP D. pulex genetic background, some CP D. pulex 135 isolates lost the capability of producing males because they carry an introgressed D. pulicaria 136 haplotype at the sex determination loci (Ye et al. 2019). Most likely, because of the genetic 137 incompatibility between the D. pulex genetic background and D. pulicaria alleles, the sex 138 determination loci in these individuals do not sufficiently respond to environmental cues for 139 initiating the male developmental program in embryos, thus losing the capability of producing 140 male progenies (Ye et al. 2019).

141 Building upon this rich biological background of the Daphnia system, this study 142 examines the gene expression of females at the early stage of resting embryo production (Figure 143 1) to identify the genetic signatures underlying the $\mathrm{CP}$ to $\mathrm{OP}$ transition. We specifically address 144 (1) the genome-wide expression changes in hybrid OP Daphnia in comparison to their parental 145 species and (2) whether aberrant expression pattern affects meiosis and cell cycle (e.g., anaphase 146 promotion complex) in OP individuals and how that may play a role in the origin of obligate 147 parthenogenesis.

\section{Materials and Methods}

149 RNA-seq experiment of females at early resting embryo production 
150 Whole-body tissues of mature females at the early stage of resting egg production were collected 151 for 5 OP isolates (Maine344-1, MC08, K09, DB4-4, Maine348-1), 2 CP D. pulex (POVI4, 152 PA42), and 2 D. pulicaria (AroMoose, LK16) isolates, respectively. These isolates were 153 collected from different geographic locations (Supplementary Table S1). Each isolate has been 154 maintained in lab conditions as an asexual mass culture, which was initiated from a single 155 asexually reproducing female. As gene expression is greatly affected by environmental 156 conditions and by maternal effects, the experimental animals of each isolate were maintained for 157 two asexual generations under $18^{\circ} \mathrm{C}$, and 12:12 (light:dark hours) photoperiod and were fed with 158 the same amount of green algae Scenedesmus obliquus as food.

The asexually produced females of the second generations constitute our focal 160 experimental animals. As these animals became mature, we examined these animals daily to 161 search for females engaging in resting egg production. As female Daphnia starts resting egg 162 production through normal meiosis in a CP isolate or parthenogenesis in an OP isolate, its ovary 163 shows a characteristic milky color and smooth texture (Hiruta and Tochinai 2014) in contrast to 164 the bulky and dark ovary in females engaging in the parthenogenetic reproduction of directly 165 developing offspring (Figure 1). In 1-2 days, an ephippium will start to form in the back of the 166 female due to carapace modification while the ovary continues to develop and become bigger. 167 For the RNA-seq experiments, we collected animals showing the characteristics of early ovary 168 development without sign of ephippium formation, which is what we defined as early resting egg 169 production. Three biological replicates of females in early resting egg production (20-30 170 individuals) for each Daphnia isolate were collected.

171 For each replicate, the RNA was extracted using the Zymo Insect RNA kit (Zymo 172 Research). The extracted RNA was prepared for short-read sequencing library construction using 173 the NEB NextUltra RNA-seq kit (New England Biolabs). The constructed libraries were 174 sequenced with 150bp paired-end reads on an Illumina HiSeq 2500 and 6000 sequencing 175 platforms. The raw DNA sequence data for this work has been deposited at NCBI SRA under 176 PRJNA726725.

177 Inheritance mode of gene expression in OP hybrids

178 As the parental gene regulatory elements coexist in the OP hybrids, their interaction determines 179 the expression of genes under control. As we hypothesized that the under-expression of genes 
180 involved in meiosis and cell cycle is key to the origin of obligate parthenogenesis, we compared

181 the expression level of each gene in the OP hybrids to that of the two CP genotypes

182 representative of each parental lineage (see below). By doing so, we can classify the gene

183 expression inheritance mode of each gene using the following set of rules (Figure 2A).

184 A gene is considered underdominant if its expression in the OP hybrid is significantly

185 lower than either parental species, whereas a gene is considered overdominant if its expression is

186 higher than either parental species. If the expression of a gene in OP is the same as one parent

187 but significantly different from the other, the inheritance is dominated by one parent, i.e., $D$.

188 pulex- or D. pulicaria-dominant. If a gene in OP is expressed at an intermediate level relative to

189 the parentals (i.e., higher than one parental but lower than the other), this gene is considered

190 additive. Lastly, if the expression of a gene in OP is found the same as both parental species, this

191 gene is considered conservative. We consider underdominance and overdominance as

192 misregulated gene expression.

193 To quantify the gene expression differences between the OP isolates and parental species,

194 the raw reads of these isolates were mapped to the D. pulex reference assembly (Ye et al. 2017)

195 using the software STAR (Dobin et al. 2013). As we also planned to do allele-specific analysis

196 (see below) to understand regulatory divergence between parental species, the mapping

197 parameters followed recommendations of Stevenson et al. (2013) for allele-specific RNA-seq

198 analysis using a single reference genome assembly. For all parental species isolates and OP

199 hybrids only the uniquely mapped reads were retained for quantifying gene expression levels.

200 The amount of sequence reads overlapping the gene body of each expressed gene was calculated

201 using the program featureCounts (Liao et al. 2014).

202 We used the program DESeq2 (Love et al. 2014) to obtain the normalized expression

203 level of each expressed gene in OP hybrids and parental isolates using the median of ratios

204 method. For each gene, we performed pairwise differential expression tests (i.e., Wald test)

205 among one OP hybrid, D. pulex, and D. pulicaria. Each OP hybrid was individually compared

206 with the parental species, and the parental species were represented by all the biological

207 replicates of the two representative isolates (e.g., D. pulex is represented by the biological

208 replicates of POVI4 and PA42). The p-values for differential expression test were corrected for a

209 false discovery rate of 0.05 using the Benjamini-Hochberg method. 
A gene's hybrid expression pattern in an OP hybrid can be delineated if at least two of

211 the three pairwise comparisons are statistically significant. Based on the outcome of these

212 comparisons, we categorized the inheritance mode of genes in each OP hybrid following the

213 rules outlined above (Figure 2A). However, for genes with only one statistically significant

214 comparison, we were not able to establish an expression mode and labelled them as ambiguous.

KEGG (Kyoto Encyclopedia of Genes and Genomes) pathway and GO term analysis

217 To identify the functional implications of misregulated gene expression (i.e., underdominance 218 and overdominance), we performed a few analyses using annotated KEGG pathways. After 219 mapping Daphnia genes into KEGG pathways using the pathway reconstruction tool 220 (https://www.genome.jp/kegg/tool/map_pathway.html), we mapped underdominant and 221 overdominant genes into KEGG pathways in each OP isolate. Within each OP isolate, we 222 calculated a underdominant/overdominant index for each pathway as the ratio of 223 underdominant/overdominant genes. We ranked the underdominant/overdominant index for 224 pathways within each OP isolate and identified the common pathways that were ranked in the 225 top 5 percentile in each OP isolate to understand which pathways were most strongly impacted 226 by misregulation.

227 We also performed KEGG pathway enrichment analysis and GO (Gene Ontology) term 228 enrichment analysis using the genes that were identified to be underdominant or overdominant in 229 all OP isolates to understand which cellular pathways and functions are disproportionately 230 affected by misregulation. To alleviate the concern that our sampling of the parental species did 231 not cover the entire expression landscape (e.g., some under- and overdominant genes may be 232 false positives), we further selected the underdominant and overdominant genes that were not 233 differentially expressed within either parental species or between the parental species. We 234 identified the differentially expressed genes within the parental D. pulex (PA42 vs POVI4) and 235 within D. pulicaria (LK16 vs AroMoose) using DESeq2 (7739 genes in total). Differentially 236 expressed genes (4294 genes) were also identified between the parental D. pulex and D.

237 pulicaria in DESeq2 using all biological replicates. The final set of underdominant and 238 overdominant genes that went into the KEGG and GO analyses did not vary in expression at 239 within- and between-species level, indicating the importance of their conserved expression level 240 in relation to reproduction in Daphnia. 
The GO enrichment analysis was performed in the topGO package (Alexa and

243 Rahnenfuhrer 2019), whereas the KEGG pathway enrichment analysis was done using a

244 hypergeometric test with a custom R script.

\section{Results}

246 Inheritance mode of gene expression in OP hybrids

247 We compared the transcript abundance of each expressed gene in OP hybrids to the two parental

248 species to examine its expression inheritance mode. Not surprisingly, conserved expression is the

249 most abundant mode in all OP hybrids, ranging between 33-56\% among all isolates (Figure 2B).

250 We noted that in the less-well sequenced samples (i.e., MC08 and Main3441), conserved

251 expression is much higher (54\% and 56\% in MC08 and M3441, respectively) than in the samples

252 of higher sequence coverage (33-38\% in M348, K09, DB4), presumably because low sequencing

253 depth reduced our power to detect differential expression in many genes between parental

254 species and OP hybrids. Thus, we suggest that the better-sequenced samples (i.e., M348, K09,

255 DB4) most likely represent an unbiased picture of the inheritance mode of gene expression in OP

256 isolates, whereas in the less-well sequenced samples the proportion of conserved expression is

257 likely inflated.

A striking pattern in the well-sequenced samples (i.e., M348, K09, DB4) is that underdominance and overdominance is the two most abundant expression mode (Figure 2B),

260 with overdominance ranging between 23-25\% and underdominance between 21-23\%. Therefore,

261 misregulated genes (underdominance and overdominance combined) constitute $45-48 \%$ of the 262 genes. In the less-well sequenced samples, overdominance ranged between 11-12\% and

263 underdominance between 9-10\%. However, across the board overdominance is consistently 2-3\%

264 higher than underdominance in each OP isolate.

265 Regarding the other expression modes, additive genes make up 3-9\% of the total genes in 266 all the samples. More interestingly, the percentage of D. pulicaria-dominant genes (9-13\%) is at 267 least two times greater than that of D. pulex-dominant genes (3-5\%) in each OP hybrid, 268 suggesting more genes resembling the expression pattern in D. pulicaria than in D. pulex. This is 269 likely because more regulatory elements of $D$. pulicaria show dominance over regulatory 270 elements of $D$. pulex. 
272 For each OP isolate, we calculated the ratio of UD and OD genes (i.e., UD and OD index) for 273 each KEGG pathway. This index indicates a possible functional link between misregulated genes

274 and obligate parthenogenesis in Daphnia. Notably, we found that the KEGG pathways with a 275 UD index in the top 5 percentile among all 5 OP isolates (Figure 3A) were Cell Cycle and 276 Meiosis, suggesting the under-expression of genes in Cell Cycle and Meiosis pathways are key 277 to the origin of obligate parthenogenesis. Pathways involved in embryo development (i.e., 278 Hedgehog signaling Pathway, P53 signaling pathway) were also among the top ranked pathways with regards to UD index, although they were only shared by 4 of the 5 OP isolates (Figure 3A).

On the other hand, KEGG pathways of OD index values in the top 5 percentile (Figure

3B) were all associated with biosynthesis and metabolism (e.g., Galactose metabolism, Histidine metabolism, Biosynthesis of unsaturated amino acids) with the exception of EGFR tyrosine

283 kinase inhibitor resistance, although it should be noted that these pathways were shared by only 4

284 of the 5 OP isolates.

\section{KEGG pathway enrichment analysis}

286 Among genes that are UD (531 genes) or OD (569 genes) in all 5 OP isolates (for gene lists see

287 Supplementary Files 1 and 2), we further selected the genes that have no significant expression 288 variation at within and between species level (for gene lists see Supplementary Files 3 and 4) and 289 used these genes (215 and 265 genes for UD and OD genes, respectively) to perform KEGG 290 pathway enrichment analysis. These gene sets have been mapped to KEGG pathways (see 291 Supplementary Files 5 and 6 for interactive html files) using the software GAEV (Huynh and Xu 292 2018). This analysis revealed that UD genes were enriched in six pathways related to cell cycle 293 meiosis, and oocyte development (hypergeometric test $\mathrm{p}<0.05$ with false discovery rate of 0.05),

294 which are Cell Cycle, Meiosis-yeast, Cell Cycle-yeast, Oocyte Meiosis, p53 signaling pathway, 295 and progesterone-mediated oocyte maturation (Figure 4A). Furthermore, OD genes were found 296 to be enriched in 23 KEGG pathways (hypergeometric test $\mathrm{p}<0.05$ with false discovery rate of 297 0.05), most of which concern metabolism and biosynthesis (Figure 4B).

\section{GO term enrichment analysis}

299 Nearly all GO terms that were enriched for the UD genes ( $p<0.01)$ were associated with cell

300 cycle, cell division, cell cycle transition, chromosome segregation, and spindle checkpoint 301 assembly (Supplementary Table S2). For example, the top five significant GO terms enriched 
302 for UD genes were "regulation of mitotic cell cycle", "cell cycle", "regulation of cell cycle

303 process", "mitotic cell cycle process", and "mitotic cell cycle". Many GO terms concerning cell

304 cycle transition, chromosome segregation, chromatid separation, spindle assembly checkpoint

305 also showed significant enrichment. On the other hand, the majority of GO terms enriched for

306 OD genes were associated with cell metabolism and biosynthesis (Supplementary Table S3),

307 with a notable exception of the top three significant terms concerning protein and macromolecule

308 glycosylation.

309 Expression pattern of UD and OD genes

310 Comparing the transcript abundance of UD and OD genes in each OP isolate to the two parental

311 species, we found that the mean $\log 2$-fold expression change of UD genes across five OP isolates

312 is -2.29 , whereas the mean $\log 2$-fold over-expression of OD genes is 1.93 (Figure 5A). The

313 absolute change of UD gene is significantly larger than that of OD genes (two sample t-test, p < $\left.3142 \times 10^{-16}\right)$.

315 As the misregulated genes in meiosis and cell cycle pathways were predominantly UD,

316 We identified several UD genes that are shared by all OP isolates and do now show expression

317 variation at both within and between species. Their $\log 2$-fold under-expression relative to the

318 parental species ranged between -0.5 and -4.5 (Figure 5B) in OP isolates. Many of these genes

319 are key players in the anaphase promotion complex (e.g., Cdc20), spindle checkpoint assembly

320 (e.g., Mad2), and cell cycle control (e.g., cell cyclin B).

322 Discussion

323 Examining gene expression in obligate parthenogens of hybrid origin in comparison with sexual

324 parental species is an effective approach to understanding how expression changes and 325 interspecific genetic incompatibility may play a role in the origin of asexuality. Indeed, our 326 analyses of the gene expression changes in OP Daphnia relative to the CP parental species, D. 327 pulex and D. pulicaria, revealed several important genetic modifications likely involved in the 328 origin of obligate parthenogenesis.

329 First, misregulated gene expression (i.e., underdominant and overdominant combined) is 330 abundant in OP Daphnia (i.e., up to 45-48\%), while conserved expression has the highest 331 number of genes out of any single inheritance mode of expression (Figure 2B). Moreover, the 332 number of overdominant genes in OP Daphnia is consistently 2-3\% higher than that of 
333 underdominant genes in all the examined samples. Clearly, compared to either parental species,

334 OP Daphnia harbors genes with significantly altered expression patterns. This stands in stark

335 contrast with the observation in the hybrid genome of asexual Cobitis loaches, where dominance

336 by one parental species accounts for the expression in 83-89\% genes and mis-regulation appears

337 to be rare (Bartos et al. 2019). Therefore, it remains to be seen how the amount of misregulated

338 genes in Daphnia compares to other hybrid obligate parthenogens and whether there are

339 consistently more overdominant vs. underdominant genes because this kind of data is lacking

340 from other obligate parthenogens.

341 Second, our analyses demonstrate that the under-expression of meiosis and cell-cycle

342 genes and over-expression of metabolic genes is a key genomic signature of parthenogenesis in

343 Daphnia. This is manifested in the significant enrichment of underdominant genes in meiosis and

344 cell-cycle pathways and GO terms and enrichment of overdominant genes in metabolic pathways

345 and GO terms in the early resting egg production stage of OP Daphnia. It should be noted that

346 the set of overdominant and underdominant genes used in the KEGG pathway and GO term

347 analyses are very conservative because we kept only genes that did not have variable expression

348 at within and between species levels. Although one may argue that we only sampled two

349 genotypes of each parental species, these overdominant and underdominant genes having

350 conserved expression at within- and between-species level strongly suggests that these genes

351 would most likely remain to be identified as underdominant or overdominant when more

352 parental genotypes are included for analysis.

353 This transcriptomic signature is not only a characteristic of early resting egg production

354 through parthenogenesis in OP hybrids, but also identified in the females at early stage of

355 asexually producing directly developing embryos in CP D. pulex and CP D. pulicaria when

356 compared to the early stage of meiotically producing haploid eggs (Huynh et al. unpublished

357 data). We therefore hypothesize that the under-expression of meiosis and cell-cycle genes plays

358 an important role in the origin of obligate parthenogenesis in hybrid OP Daphnia. On the other

359 hand, the over-expression of metabolic genes may be a trigger of parthenogenetic reproductive

360 pathways or simply a response to environmental cues. Testing these hypotheses would entail

361 detailed molecular genetic analyses of these genes in relation to obligately parthenogenetic

362 reproduction. We suspect that the causal factors would likely involve only a few key under- and 
363 overdominant genes affecting key pathways, whereas many of the other identified genes could be

364 of secondary consequences.

365 One noteworthy underdominant gene (i.e., Cdc20) has also been identified as candidate 366 genes involved in the origin of obligate parthenogenesis in Daphnia (Eads et al. 2012; Xu et al. 367 2015), whereas another underdominant gene Mad2 is an important member of the spindle 368 checkpoint assembly. As the parthenogenetic production of resting embryos in OP Daphnia is 369 most likely achieved through a modified form of meiosis (Zaffagnini and Sabelli 1972) where 370 segregation is suppressed and anaphase I does not lead to cytokinesis, the under-expression of 371 these genes in OP Daphnia may very well be essential to these modifications. Cdc20 is a key cell 372 cycle regulator ( $\mathrm{Yu}$ 2007) and its under-expression may play a more significant role than other 373 genes. Although our results are based on RNA-seq of whole-body tissue, this idea draws support 374 from emerging molecular evidence. For example, the under-expression of Cdc20 in mouse 375 results in failed segregation of chromosomes in oocytes (Jin et al. 2010). This idea is worth of 376 further investigation at the tissue-specific and cellular level.

Third, the genetic mechanisms underlying the misregulation of the identified genes remain to be examined. Identifying the genetic loci and variants regulating the expression of these genes through eQTL (expression quantitative trait loci) mapping would be helpful for us to

380 answer questions such as whether the misregulation is a direct consequence of the interaction 381 between the introgressed $D$. pulicaria alleles with $D$. pulex genetic background.

382 Lastly, we emphasize that OP in D. pulex is most likely a polygenic trait (Xu et al. 383 2015). There are other genetic components involved in OP that need to be further investigated, 384 e.g., the suppression of recombination during homologous pairing and the bypass of oocyte 385 development arrest without fertilization. Hopefully, future studies can identify the possible 386 candidate genes. Most importantly, we believe that molecular functional characterization of the 387 roles of the identified candidate genes in OP is much needed, which can provide a novel 388 perspective on the origin of parthenogenesis and on the genetic incompatibility in closely related 389 species. 


\section{Acknowledgements}

392 We thank A. Hall for his help with the experiments. This work is supported by NIH MIRA grant

393 R35GM133730 to SX. This research used computational resources supported by the National

394 Science Foundation under Grant Nos. DBI-1062432 2011, ABI-1458641 2015, and ABI-

39517599062018 to Indiana University. Any opinions, findings, and conclusions or

396 recommendations expressed in this material are those of the authors and do not necessarily

397 reflect the views of the National Science Foundation, the National Center for Genome Analysis

398 Support, or Indiana University.

399 Conflicts of interests

400 The authors declare no conflicts of interests. 


\section{References}

402

403

404

405

406

407

408

409

410

411

412

413

414

415

416

417

418

419

420

421

422

423

424

425

426

427

428

429

430

431

Alexa, A. and J. Rahnenfuhrer. 2019. topGO: Enrichment Analysis for Gene Ontology. topGO: Enrichment Analysis for Gene Ontology.

Avise, J. C. 2015. Evolutionary perspectives on clonal reproduction in vertebrate animals. Proc. Natl. Acad. Sci. USA 112:8867-8873.

Barbash, D. A., D. F. Siino, A. M. Tarone, and J. Roote. 2003. A rapidly evolving MYB-related protein causes species isolation in Drosophila. Proc. Natl. Acad. Sci. USA 100:53025307.

Bartos, O., J. Roslein, J. Kotusz, J. Paces, L. Pekarik, M. Petrtyl, K. Halacka, E. Stefkova Kasparova, J. Mendel, A. Boron, D. Juchno, A. Leska, O. Jablonska, V. Benes, M. Sidova, and K. Janko. 2019. The Legacy of Sexual Ancestors in Phenotypic Variability, Gene Expression, and Homoeolog Regulation of Asexual Hybrids and Polyploids. Mol. Biol. Evol. 36:1902-1920.

Bell, G. 1982. The masterpiece of nature: the evolution and genetics of sexuality. Kluwer Academic Publishers.

Benjamini, Y. and Y. Hochberg. 1995. Controlling the False Discovery Rate: A Practical and Powerful Approach to Multiple Testing. Controlling the False Discovery Rate: A Practical and Powerful Approach to Multiple Testing 57:289-300.

Brandlova, J., Z. Brandl, and C. H. Fernando. 1972. The Cladocera of Ontario with remarks on some species and distribution. Can. J. Zool. 50:1373-1403.

Caceres, C. E. and A. J. Tessier. 2004a. Incidence of diapause varies among populations of Daphnia pulicaria. Oecologia 141:425-431.

Caceres, C. E. and A. J. Tessier. 2004b. To sink or swim: Variable diapause strategies among Daphnia species. Limnol. Oceanogr. 49:1333-1340.

Chin, T. A., C. E. Caceres, and M. E. Cristescu. 2019. The evolution of reproductive isolation in Daphnia. BMC Evol. Biol. 19:216.

Colbourne, J. K. and P. D. N. Hebert. 1996. The systematics of North American Daphnia (Crustacea: Anomopoda): A molecular phylogenetic approach. Philos. Trans. R. Soc. Lond., B, Biol. Sci. 351:349-360.

Colegrave, N. 2002. Sex releases the speed limit on evolution. Nature 420:664-666. 
Colegrave, N., O. Kaltz, and G. Bell. 2002. The ecology and genetics of fitness in Chlamydomonas. VIII. The dynamics of adaptation to novel environments after a single episode of sex. Evolution 56:14-21.

Cooper, K. F. and R. Strich. 2011. Meiotic control of the APC/C: similarities \& differences from mitosis. Meiotic control of the APC/C: similarities \& differences from mitosis 6:16.

Cooper, T. F. 2007. Recombination speeds adaptation by reducing competition between beneficial mutations in populations of Escherichia coli. PLoS Biol. 5:1899-1905.

Cristescu, M. E., A. Constantin, D. G. Bock, C. E. Caceres, and T. J. Crease. 2012. Speciation with gene flow and the genetics of habitat transitions. Mol. Ecol. 21:1411-1422.

Deng, H. W. 1997. Photoperiodic response of sexual reproduction in the Daphnia pulex group is reversed in two distinct habitats. Limnol. Oceanogr. 42:609-611.

Dobin, A., C. A. Davis, F. Schlesinger, J. Drenkow, C. Zaleski, S. Jha, P. Batut, M. Chaisson, and T. R. Gingeras. 2013. STAR: ultrafast universal RNA-seq aligner. Bioinformatics 29:15-21.

Dobzhansky, T. 1937. Genetics and the origin of species. Columbia Univ. Press, New York,.

Dudycha, J. L. 2004. Mortality dynamics of Daphnia in contrasting habitats and their role in ecological divergence. Freshw. Biol. 49:505-514.

Dudycha, J. L. and A. J. Tessier. 1999. Natural genetic variation of life span, reproduction, and juvenile growth in Daphnia. Evolution 53:1744-1756.

Eads, B. D., D. Tsuchiya, J. Andrews, M. Lynch, and M. E. Zolan. 2012. The spread of a transposon insertion in Rec 8 is associated with obligate asexuality in Daphnia. Proc. Natl. Acad. Sci. USA 109:858-863.

Felsenstein, J. 1974. The evolutionary advantage of recombination. Genetics 78:737-756.

Gabriel, W., M. Lynch, and R. Burger. 1993. Mullers Ratchet and Mutational Meltdowns. Evolution 47:1744-1757.

Gallot, A., S. Shigenobu, T. Hashiyama, S. Jaubert-Possamai, and D. Tagu. 2012. Sexual and asexual oogenesis require the expression of unique and shared sets of genes in the insect Acyrthosiphon pisum. BMC Genomics 13.

Goddard, M. R., H. Charles, J. Godfray, and A. Burt. 2005. Sex increases the efficacy of natural selection in experimental yeast populations. Nature 434:636-640. 
Gorbsky, G. J. 2015. The spindle checkpoint and chromosome segregation in meiosis. FEBS J. 282:2471-2487.

Hanson, S. J., A. M. Schurko, B. Hecox-Lea, D. B. M. Welch, C. P. Stelzer, and J. M. Logsdon. 2013. Inventory and phylogenetic analysis of meiotic genes in monogonont rotifers. J. Hered. 104:357-370.

Hebert, P. D. N., S. S. Schwartz, R. D. Ward, and T. L. Finston. 1993. Macrogeographic patterns of breeding system diversity in the Daphnia pulex group. 1. breeding systems of Canadian populations. Heredity 70:148-161.

Heier, C. R. and J. L. Dudycha. 2009. Ecological speciation in a cyclic parthenogen: sexual capability of experimental hybrids between Daphnia pulex and Daphnia pulicaria. Limnol. Oceanogr. 54:492-502.

Hiruta, C., C. Nishida, and S. Tochinai. 2010. Abortive meiosis in the oogenesis of parthenogenetic Daphnia pulex. Chromosome Res. 18:833-840.

Hiruta, C. and S. Tochinai. 2014. Formation and structure of the ephippium (resting egg case) in relation to molting and egg laying in the water flea Daphnia pulex De Geer (Cladocera: Daphniidae). J. Morphol. 275:760-767.

Hotz, H., G. Mancino, S. Bucciinnocenti, M. Ragghianti, L. Berger, and T. Uzzell. 1985. Rana ridibunda varies geographically in inducing clonal gametogenesis in interspecies hybrids. J. Exp. Zool. 236:199-210.

Huynh, T. and S. Xu. 2018. Gene Annotation Easy Viewer (GAEV): Integrating KEGG's Gene Function Annotations and Associated Molecular Pathways. Gene Annotation Easy Viewer (GAEV): Integrating KEGG's Gene Function Annotations and Associated Molecular Pathways 7:416.

Janko, K., J. Pačes, H. Wilkinson-Herbots, R. J. Costa, J. Röslein, P. Drozd, N. Iakovenko, J. Rídl, J. Kočí, R. Reifová, V. Šlechtová, and L. Choleva. 2018. Hybrid asexuality as a primary reproductive barrier: on the interconnection between asexuality and speciation. Mol. Ecol. 27:248-263.

Jin, F., M. Hamada, L. Malureanu, K. B. Jeganathan, W. Zhou, D. E. Morbeck, and J. M. van Deursen. 2010. Cdc20 is critical for meiosis $i$ and fertility of female mice. PLoS Genet. 6:e1001147.

Kaltz, O. and G. Bell. 2002. The ecology and genetics of fitness in Chlamydomonas. XII. Repeated sexual episodes increase rates of adaptation to novel environments. Evolution 56:1743-1753. 
Kearney, M., M. K. Fujita, and J. Ridenour. 2009. Lost sex in the reptiles: constraints and correlations. Pp. 447-474 in I. Schön, K. Martens, and P. van Dijk, eds. Lost Sex. Springer, Dordrecht.

Kosheleva, K. and M. M. Desai. 2018. Recombination Alters the Dynamics of Adaptation on Standing Variation in Laboratory Yeast Populations. Mol. Biol. Evol. 35:180-201.

Liao, Y., G. K. Smyth, and W. Shi. 2014. featureCounts: an efficient general purpose program for assigning sequence reads to genomic features. Bioinformatics 30:923-930.

Love, M. I., W. Huber, and S. Anders. 2014. Moderated estimation of fold change and dispersion for RNA-seq data with DESeq2. Genome Biol. 15:550.

Lynch, M., R. Burger, D. Butcher, and W. Gabriel. 1993. The mutational meltdown in asexual populations. J. Hered. 84:339-344.

Lynch, M., A. Seyfert, B. Eads, and E. Williams. 2008. Localization of the genetic determinants of meiosis suppression in Daphnia pulex. Genetics 180:317-327.

Mack, K. L. and M. W. Nachman. 2016. Gene regulation and speciation. Trends Genet. 33:68-80.

Maynard Smith, J. 1978. The evolution of sex. Cambridge University Press, Cambridge; New York.

McManus, C. J., J. D. Coolon, M. O. Duff, J. Eipper-Mains, B. R. Graveley, and P. J. Wittkopp. 2010. Regulatory divergence in Drosophila revealed by mRNA-seq. Genome Res. 20:816-825.

Millette, K. L., A. Gonzalez, and M. E. Cristescu. 2020. Breaking ecological barriers: Anthropogenic disturbance leads to habitat transitions, hybridization, and high genetic diversity. Sci. Total Environ. 740:140046.

Muller, H. 1964. The relation of recombination to mutational advance. Mutat. Res. 106:2-9.

Muller, H. J. 1940. Bearing of the Drosophila work on systematics. Pp. 185-268 in J. Huxley, ed. Bearing of the Drosophila work on systematics. Claredon Press.

Neiman, M., T. F. Sharbel, and T. Schwander. 2014. Genetic causes of transitions from sexual reproduction to asexuality in plants and animals. J. Evol. Biol. 27:1346-1359.

Omilian, A. R. and M. Lynch. 2009. Patterns of intraspecific DNA variation in the Daphnia nuclear genome. Genetics 182:325-336. 
Otto, S. P. 2009. The evolutionary enigma of sex. Am. Nat. 174:S1-S14.

525

526

527

528

529

530

531

532

533

534

535

536

537

538

539

540

541

542

543

544

545

546

547

548

549

550

551

552

Poon, A. and L. Chao. 2004. Drift increases the advantage of sex in RNA bacteriophage Phi 6. Genetics 166:19-24.

Presgraves, D. C. 2003. A fine-scale genetic analysis of hybrid incompatibilities in Drosophila. Genetics 163:955-972.

Rawson, P. D. and R. S. Burton. 2002. Functional coadaptation between cytochrome c and cytochrome c oxidase within allopatric populations of a marine copepod. Proc. Natl. Acad. Sci. USA 99:12955-12958.

Schultz, R. J. 1973. Unisexual fish: laboratory synthesis of a species. Science 179:180-181.

Simon, J. C., F. Delmotte, C. Rispe, and T. Crease. 2003. Phylogenetic relationships between parthenogens and their sexual relatives: the possible routes to parthenogenesis in animals. Biol. J. Linn. Soc. 79:151-163.

Srinivasan, D. G., A. Abdelhady, and D. L. Stern. 2014. Gene Expression Analysis of Parthenogenetic Embryonic Development of the Pea Aphid, Acyrthosiphon pisum, Suggests That Aphid Parthenogenesis Evolved from Meiotic Oogenesis. Plos One 9.

Stenberg, P. and A. Saura. 2009. Cytology of asexual animals. Pp. 63-74 in I. Schön, K. Martens, and P. van Dijk, eds. Lost Sex. Springer, Dordrecht.

Stern, D. L. and V. Orgogozo. 2008. The loci of evolution: How predictable is genetic evolution? Evolution 62:2155-2177.

Stevenson, K. R., J. D. Coolon, and P. J. Wittkopp. 2013. Sources of bias in measures of allelespecific expression derived from RNA-seq data aligned to a single reference genome. BMC Genomics 14.

White, M. J. D., N. Contreras, J. Cheney, and G. C. Webb. 1977. Cytogenetics of parthenogenetic grasshopper Warramaba (formerly Moraba) virgo and its bisexual relatives .2. hybridization studies. Chromosoma 61:127-148.

Wray, G. A. 2007. The evolutionary significance of cis-regulatory mutations. Nat. Rev. Genet. 8:206-216.

Xu, S., D. J. Innes, M. Lynch, and M. E. Cristescu. 2013. The role of hybridization in the origin and spread of asexuality in Daphnia. Mol. Ecol. 22:4549-4561. 
Xu, S., K. Spitze, M. S. Ackerman, Z. Ye, L. Bright, N. Keith, C. E. Jackson, J. R. Shaw, and M. Lynch. 2015. Hybridization and the origin of contagious asexuality in Daphnia pulex. Mol. Biol. Evol. 32:3215-3225.

556 Ye, Z., C. Molinier, C. Zhao, C. R. Haag, and M. Lynch. 2019. Genetic control of male production in Daphnia pulex. Proc. Natl. Acad. Sci. USA 116:15602-15609.

Ye, Z., S. Xu, K. Spitze, J. Asselman, X. Jiang, M. S. Ackerman, J. Lopez, B. Harker, R. T. Raborn, W. K. Thomas, J. Ramsdell, M. E. Pfrender, and M. Lynch. 2017. A new reference genome assembly for the microcrustacean Daphnia pulex. G3 (Bethesda) 7:1405-1416.

Yu, H. 2007. Cdc20: A WD40 activator for a cell cycle degradation machine. Mol. Cell 27:3-16.

Zaffagnini, F. and B. Sabelli. 1972. Karyologic observations on the maturation of the summer and winter eggs of Daphnia pulex and Daphnia middendorffiana. Chromosoma 36:193203. 
568 Figure 1. Life history of cyclical parthenogenesis (A) and obligate parthenogenesis (B) in 569 Daphnia.
A
Cyclical parthenogenesis

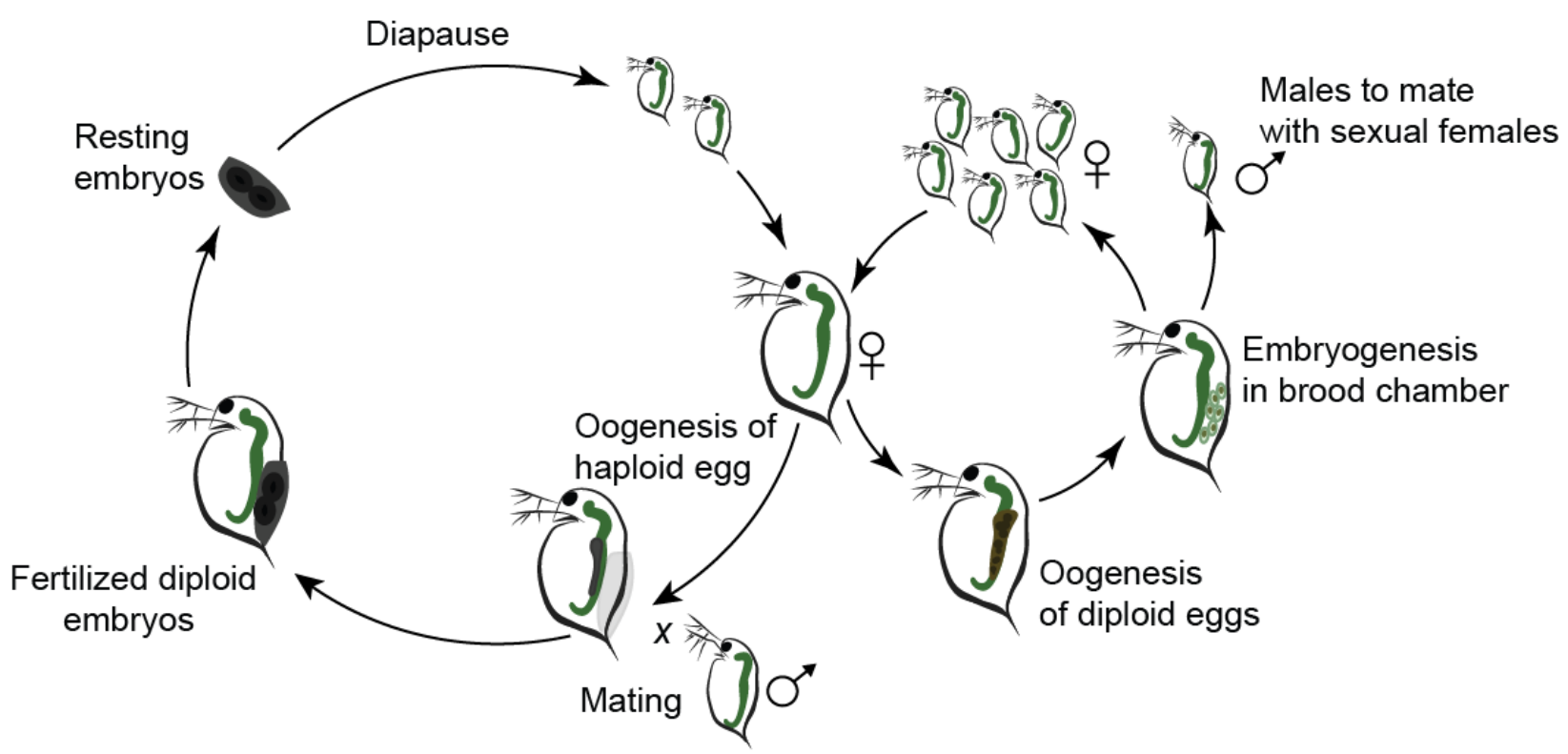

B

Obligate parthenogenesis

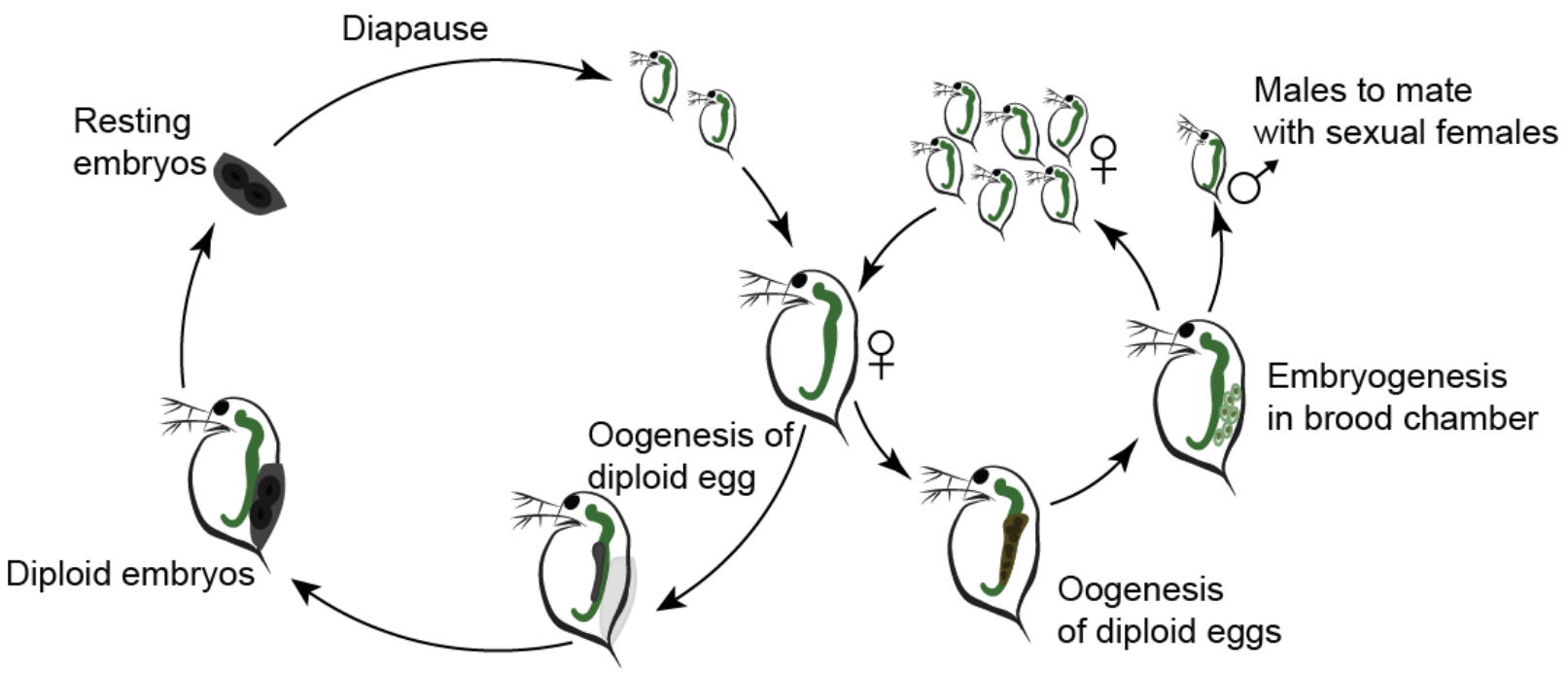


573 Figure 2. (A) Graphic summary of the criteria for classifying inheritance mode of expression

574 following McManus et al. (2010). (B) Summary of the number of genes in different inheritance

575 mode in five obligately parthenogenetic Daphnia isolates. Numbers next to each horizontal bar

576 represent the count of genes, with percentage in the parenthesis.

A
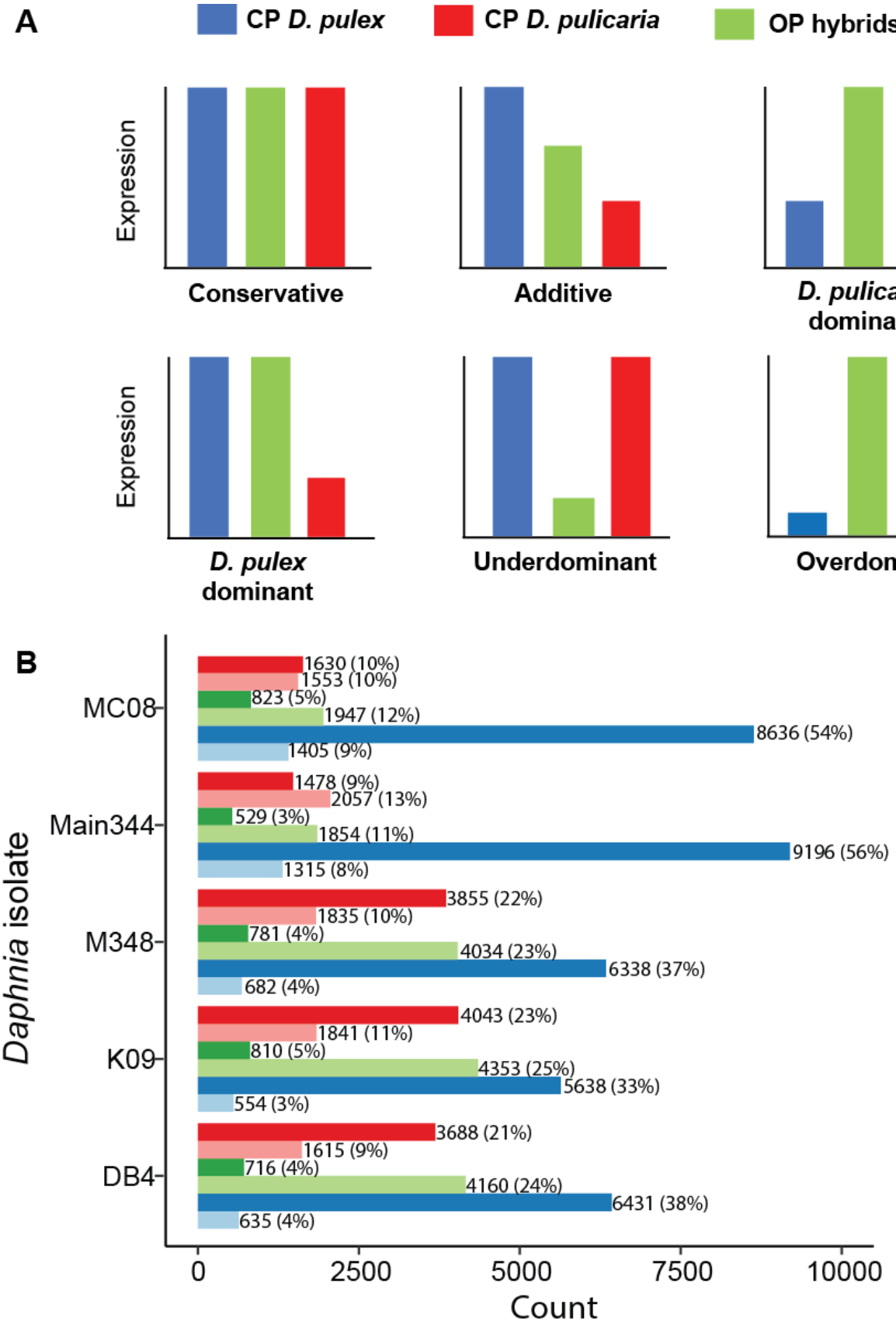

\section{OP hybrids}
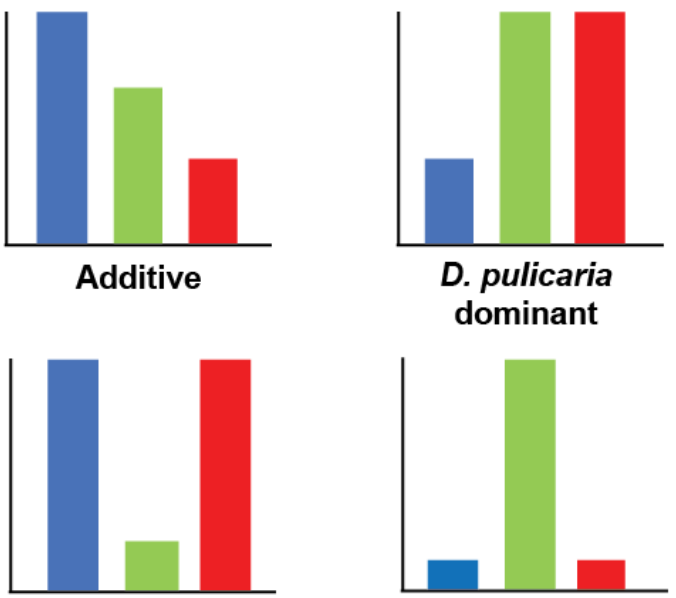

Underdominant

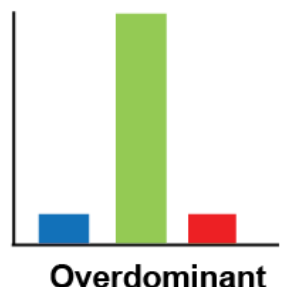

Inheritance Mode

Additive

Conservative

Overdominant

D. pulex-dominant

D. pulicaria-dominant

Underdominant 
580 Figure 3. The distribution of underdominance index (A) and overdominance index (B) for

581 KEGG pathways in obligately parthenogenetic Daphnia isolates. The vertical dashed lines

582 represent the 95 percentile in each isolate. The KEGG pathways with an index value in the top 5

583 percentile in at least 4 Daphnia isolates (pathway name - number of isolates) are listed.

584
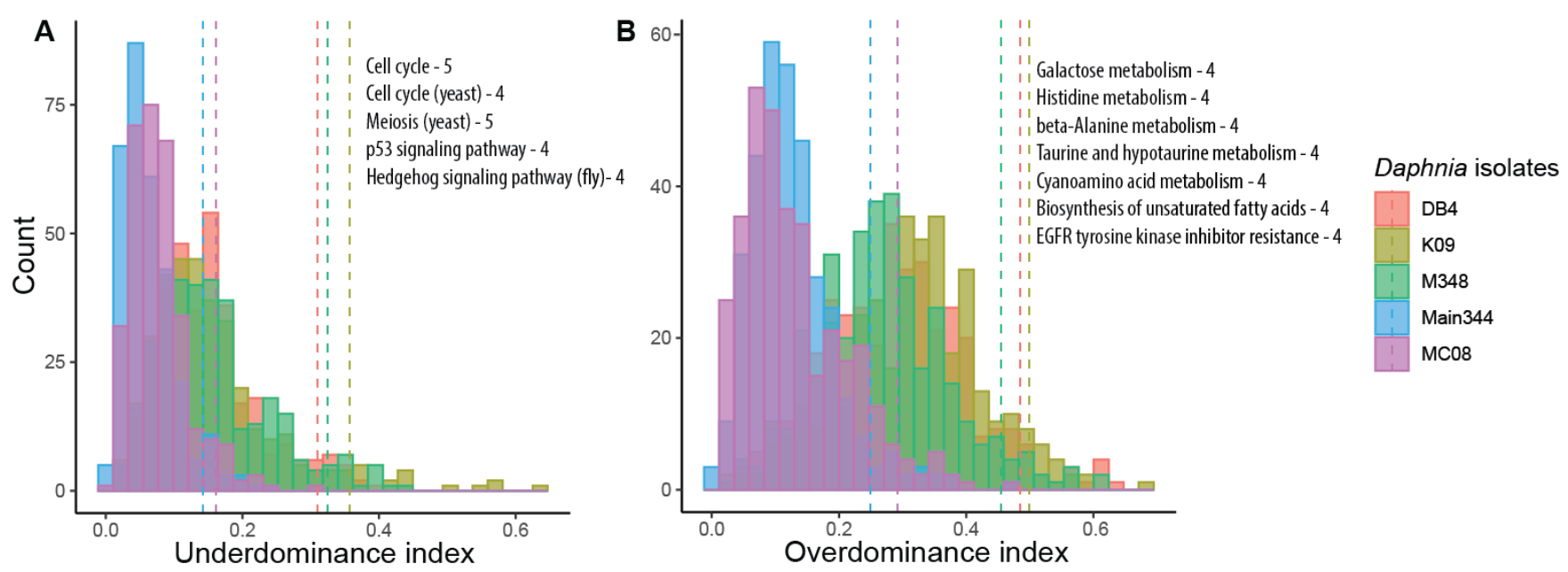
586 Figure 4. KEGG pathways enriched with underdominant (UD) genes (A) and with overdominant (OD) genes (B). UD/OD gene ratio

587 (horizontal axis) represents the proportion of UD/OD genes in the pathway. p.adjust values are hypergeometric test $\mathrm{p}$ values corrected

588 for multiple testing using Benjamini and Hochberg (1995) method.

589

A

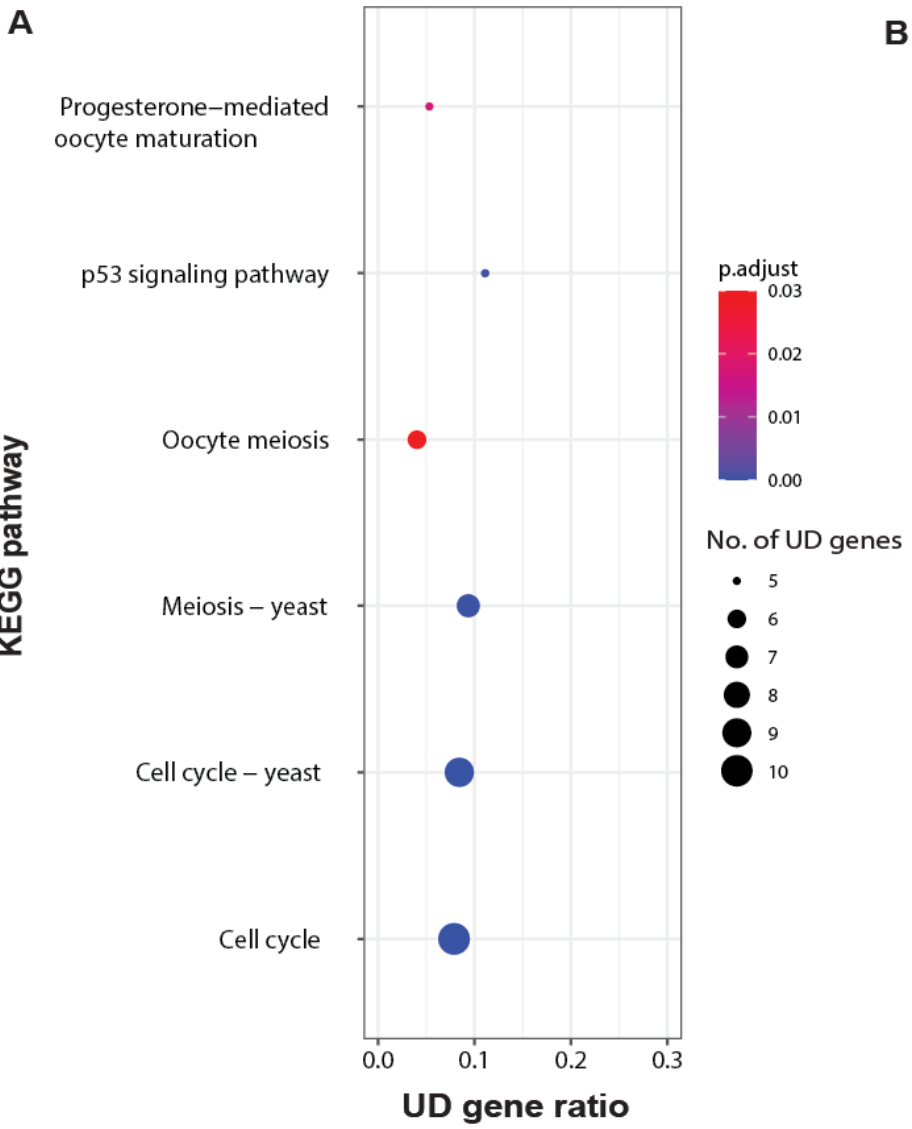

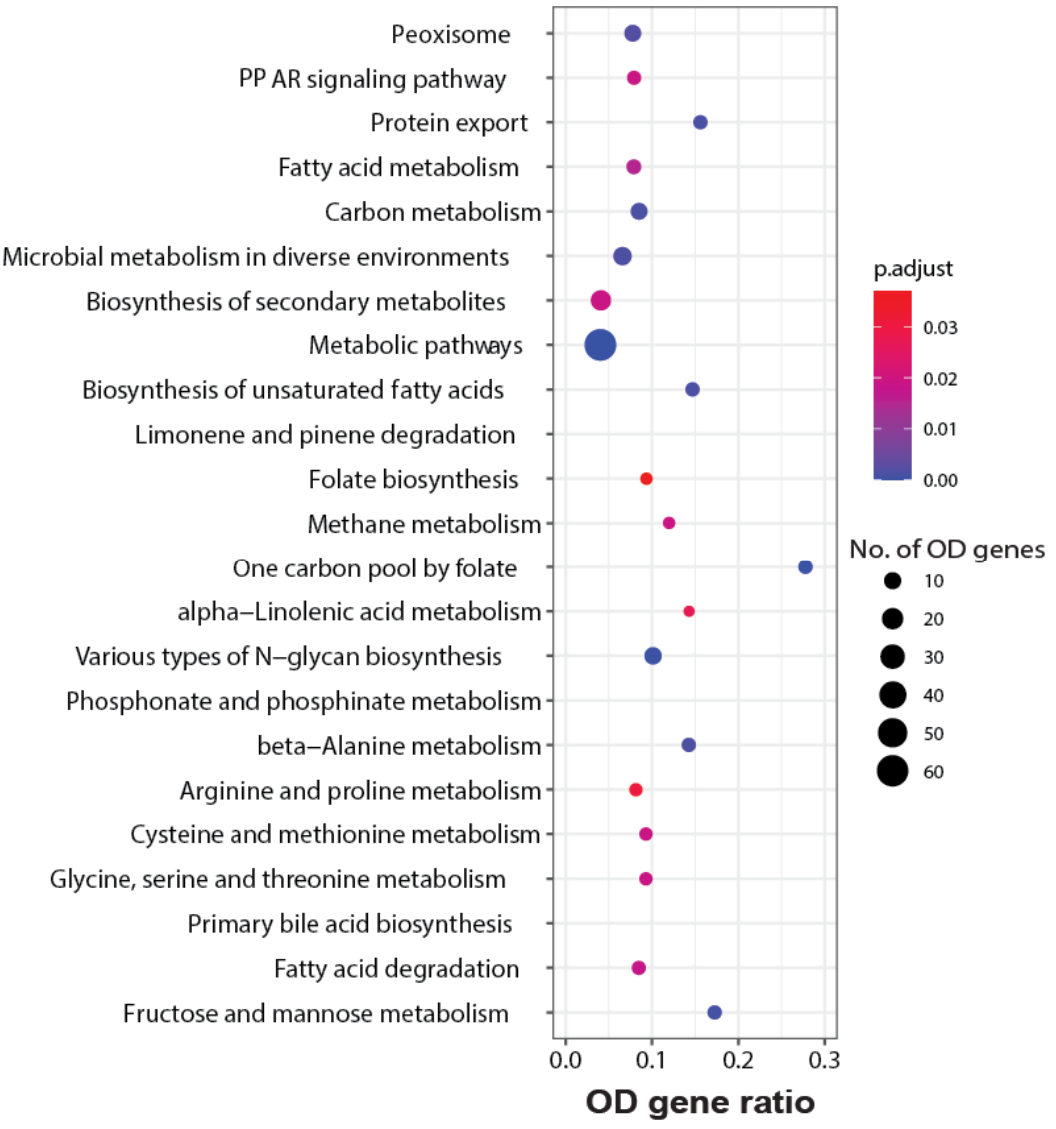


591 Figure 5. (A) Histograms of log2-fold change of underdominant (UD) and overdominant (OD)

592 genes in obligately parthenogenetic Daphnia isolates, with mean value indicated by dashed lines.

593 (B) Log2-fold expression change of meiosis and cell-cycle genes that were underdominant in all

594 examined obligately parthenogenetic Daphnia isolates.

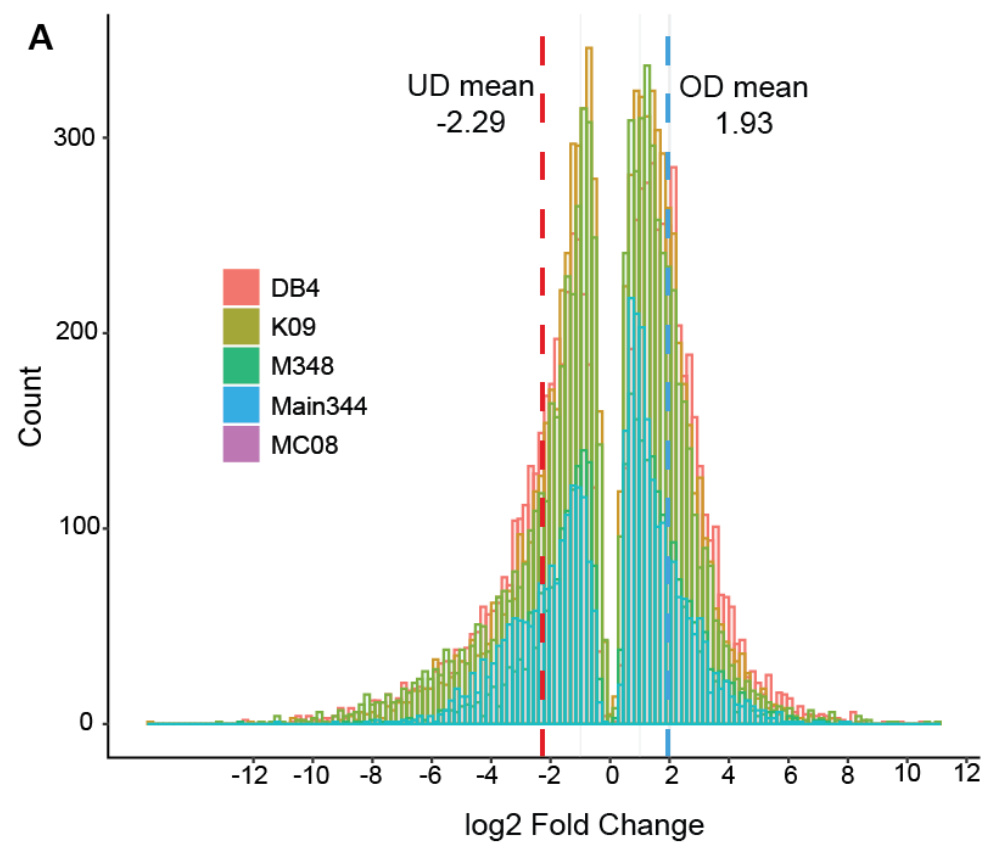

602

B

603

604

605

$0.0-$

606

607

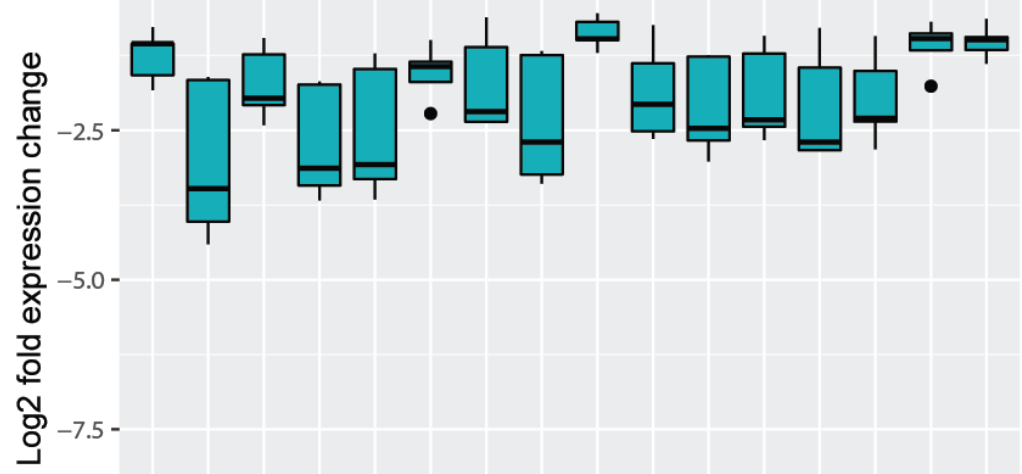

608

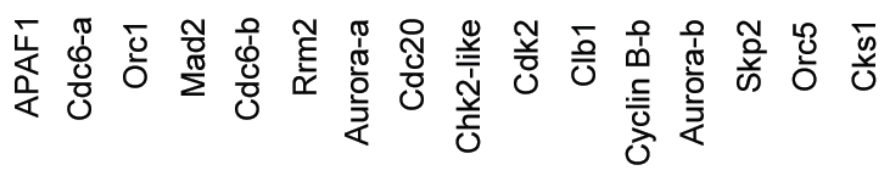

609 